\title{
Phasechronometric measuring technologies application for turning tool wear monitoring
}

\author{
Anthony Syritsky, ${ }^{1, *}$ \\ ${ }^{1}$ Bauman MSTU, Machine building technology Department, 105005 Moscow, Russia
}

\begin{abstract}
The article briefly describes the results of creating a phasechronometric system for monitoring the state of a turning tool. The realization of the method in practice is shown. The composition of the original measuring unit is described. The results of theoretical and experimental studies are presented. The working capacity of the new method in industrial conditions is proved. The proposed method makes it possible to monitor the wear of the lathe with high accuracy during machining.
\end{abstract}

In the key of manufacturing informatization it becomes an important issue to obtain information about all the key options of work metalworking machines. Lack of information and let one but very important parameter during processing will mean reducing the effect of gaining momentum convergence of information technology and metalworking [1,2]. One of such important parameters are the information about the technical condition of the machine components and the actual state of the least reliable processing element of the system - the cutting tool. It is an assessment tool wear directly in the treatment process with a view to the timely replacement and diagnosis of rolling lathes to evade the system of preventive maintenance.

In this study as a tool to obtain more information about the parameters of the work lathes considered phasechranometric method [3], which is based on the continuous measurement of the time intervals between the phases of the machine cycle or machine (Fig .1).

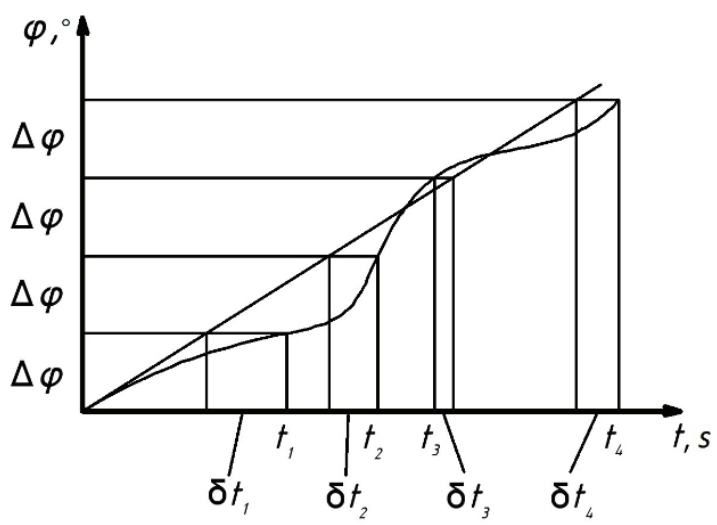

Fig. 1. Phasechronometric measurements principle.
In this method, time intervals $\delta t_{1} \ldots \delta t_{i}$, the corresponding rotation (displacement) of the vehicle chassis undergoing a cycle are measured, in this case the machine spindle at a certain angle $\Delta \varphi$ (cycle phase). Experience about phasechronometric on other machines shows that the measurement of time intervals of the machine spindle revolution in the processing phase, we can obtain information about the operation of the machine. Information on the operation of all machine components, including the state of the tool is included in the chronogram of rotation, which is a graphic reflection of the values of the time series $\delta t_{1} \ldots \delta t_{i}$ (chronogram formation circuit is shown in Figure 2). Real chronograms are shown in Figure 3-4. Figure 4 shows the chronogram for turning a new cutter. Figure 5 shows the chronogram for turning with a tool with wear $\mathrm{VB}=400 \mu \mathrm{m}$

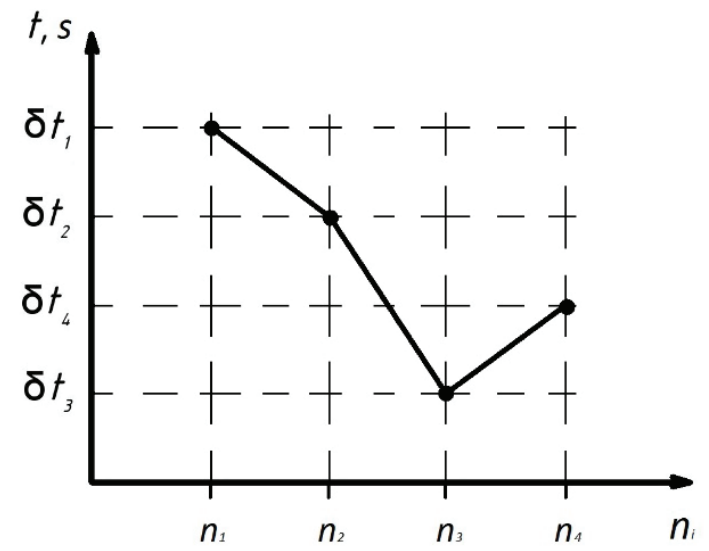

Fig. 2. Chronogram formation.

\footnotetext{
* Corresponding author: syritsky@yandex.ru
} 


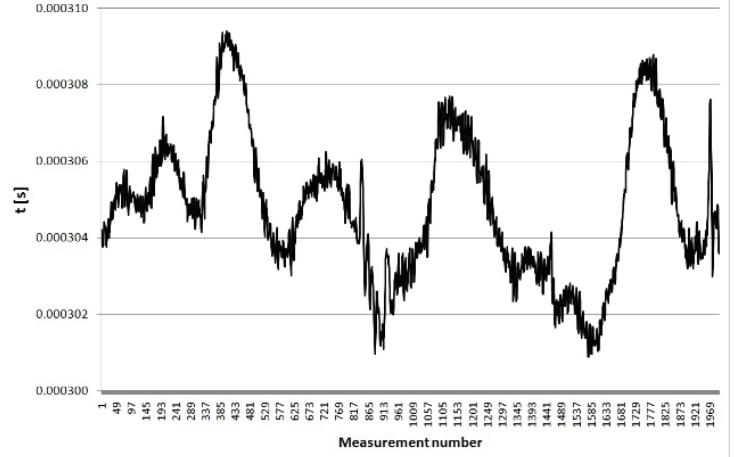

Fig. 3. Chronogram for turning a new cutter.

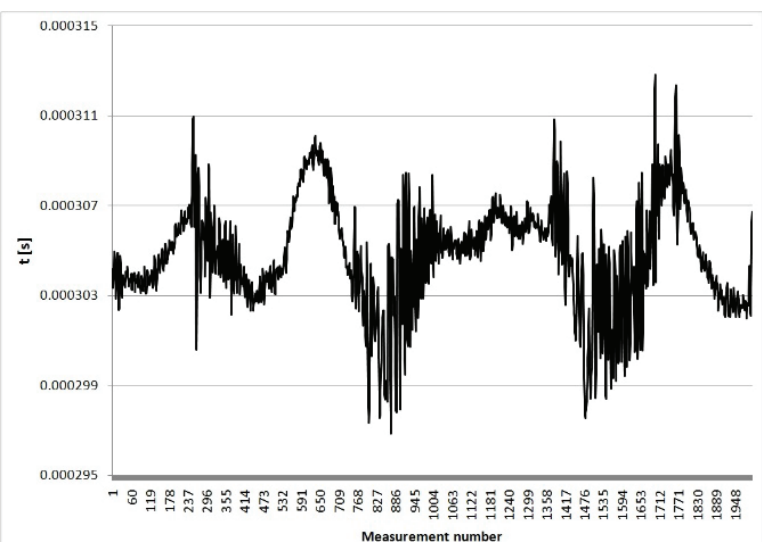

Fig. 4. Chronogram for turning with a tool with wear $\mathrm{VB}=400$ $\mu \mathrm{m}$

This approach is radically different from those that are used at present in mechanical amplitude techniques (vibration diagnostics and so on.) firstly by accuracy of the obtaining measurement information. Due to the fact that at the moment the frequency and time is possible to measure with the highest accuracy, phasechronometric systems are characterized by a relative error of measurement at the level of 10-2 - 10-4\%. Measuring channels used to date diagnostic systems do not reach this level of precision.

With regard to the lathes phasechronometric system consists of three main elements (Figure 1): a primary converter, programmable logic integrated circuit module (FPGAs) and embedded computer.

On the spindle is set as transducer or incremental encoder, or information element (disc with grooves) in conjunction with inductive or optical sensor [10,11]. An example of installing a sensor on a lathe is shown in Figures 5, 6.

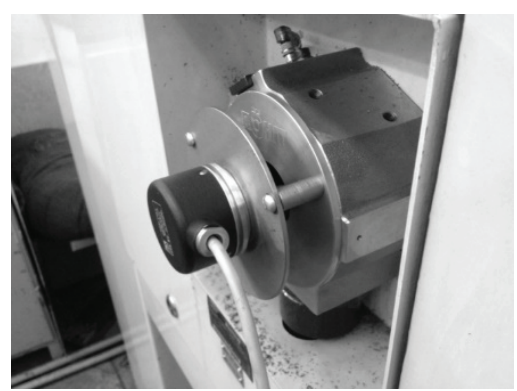

Fig. 5. General view of the angle sensor mounting

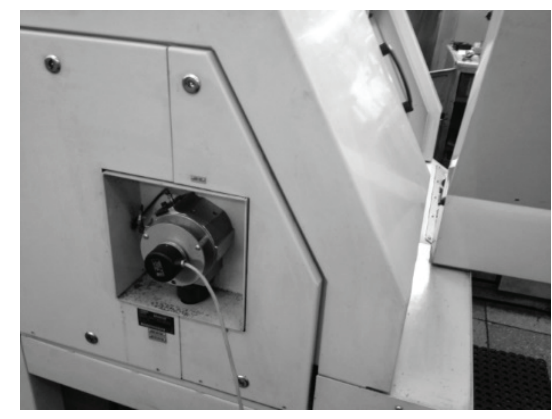

Fig. 6. Installation of the angle sensor on the lathe machine

The converter generates a sinusoidal analog signal $U$ $(\mathrm{t})$, which is then transmitted to the FPGA for processing where the signal is converted to digital form for transmission to the computer. FPGA module in turn consist of several blocks (Fig .7).

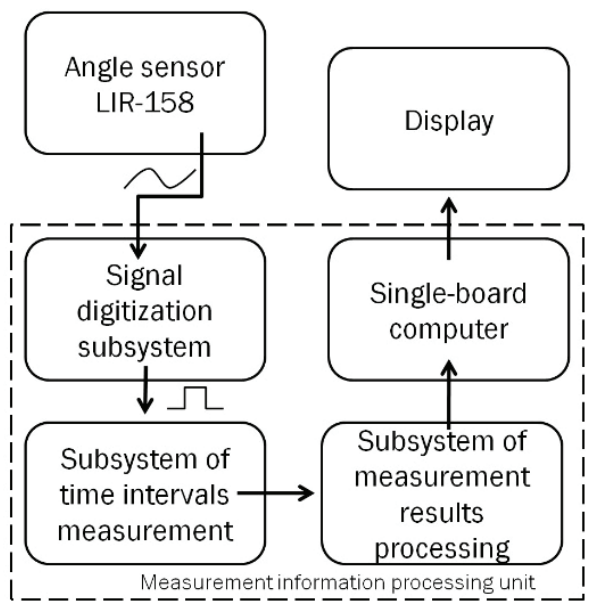

Fig. 7. Measurement unit scheme.

The output of the comparator goes to eliminating bounce circuit which registers clearly establish the level of high-frequency vibrations (bounce) during the transition from the state of the log. 0 in the log. 1 and vice versa. The basic unit of measurement system is formed by the timer and reset circuitry, and represents a real-time clock for measuring time intervals. Because of the different work time FPGA and embedded computer (FPGA works in real-time, embedded computer - no) required a buffer, which is used to synchronize threads. Through a protocol driver from the measured time intervals are generated measuring data packets which are transmitted to the embedded computer for further processing, visualization, and software compatibility with the corresponding industrial equipment. Embedded Systems is a single-board computer with Linux Lubuntu 13.04 operating system. Software platform consists of two parts: a driver for receiving and transmitting data from the bus and the user application to signal processing and imaging. Next, the data as a function of the node status information fed to the machine operator system.

Special interest in matters of the development phasechronometric system-turning tool of state of the cutting edge causes the problem of determining the blunt tool criteria. To build an effective monitoring system requires the determination of a parameter that varies over 
time with wear. That option change of the relative proportion of high-frequency oscillations in the spectrum A (f) rotation chronogram (Figure 8) was selected.

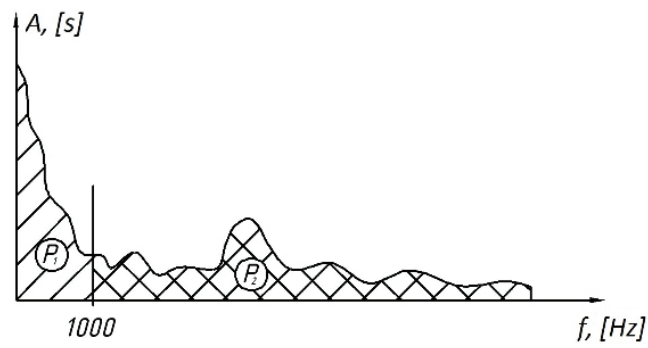

Fig. 8. The relative proportion of high-frequency oscillations in the rotation chronogram spectrum.

It is known that fluctuations of up to $1000 \mathrm{~Hz}$, for this type of machines includes information about uneven work machine motor, toothing defects, lubrication. It is known that fluctuations connected with the wear of the turning tool is high-frequency. Therefore, dividing the power spectrum of the field of high-frequency oscillations (region $\mathrm{P}_{2}$ ) to a total capacity of the oscillation spectrum (area $\mathrm{P}_{1}+$ region $\mathrm{P}_{2}$ ), you can get a percentage of $\Psi$ that increase is correlated with the wear of the cutting edge:

$$
\Psi=\frac{\mathrm{P}_{2}}{\mathrm{P}_{1}+\mathrm{P}_{2}} \cdot 100 \%
$$
$\mathrm{W}$;

where $\mathrm{P}_{1}$ - total capacity of the oscillation spectrum,

$\mathrm{P}_{2}$ - spectrum power of the field of high-frequency vibrations, W.

Experimental research has focused on the confirmation of the above hypothesis. Their implementation consisted in processing a cylindrical workpiece surface diameter of $70 \mathrm{~mm}$ made of steel 45 in the machine $16 \mathrm{~K} 20$ and recording chronometric data during cutting. Depth of cut $\mathrm{t}=1 \mathrm{~mm}$, feed $\mathrm{S}=0,2 \mathrm{~mm}$, spindle speed of the machine $\mathrm{n}=1000 \mathrm{rev} / \mathrm{min}$. Tool final state was evaluated by measuring the VB parameter on a precision video-measuring machine Mitutoyo Quick Vision Apex Pro. Flank wear was 500 micrometers. The results are presented in figure 9.

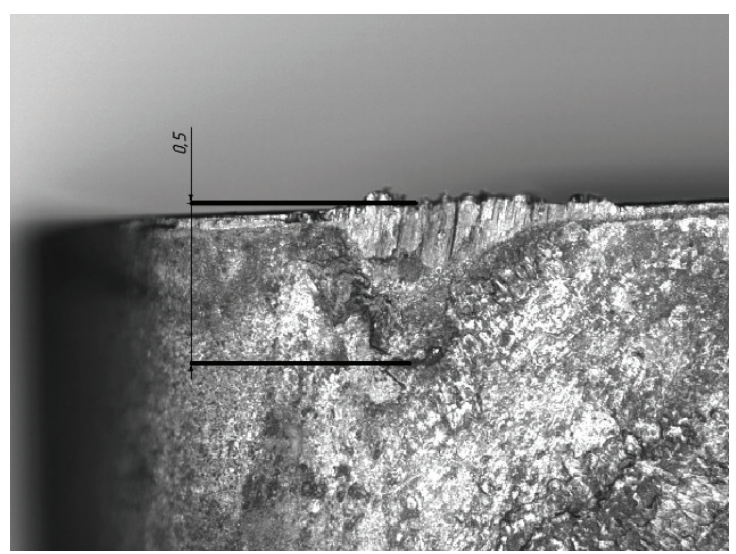

Fig. 9. Flank wear, mm.
Consistently through equal treatment intervals of time were constructed assess changes in the relative proportion of high-frequency oscillations in the spectrum chronogram $\Psi$ rotation. The results are shown in Figure 10:

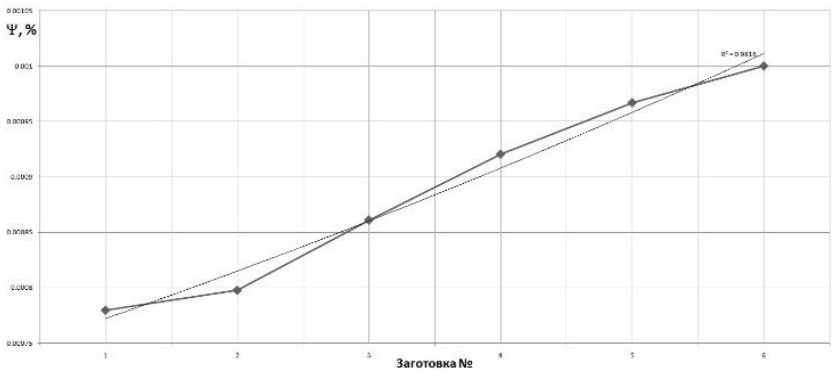

Fig. 10. Assesment of changes in the relative proportion of highfrequency vibrations in the range of rotation chronogram.

As seen in Figure 10, a straight trend line increases with increasing tool wear, so at the moment this method of processing the received measurement information can be identified as the most perspective.

Because of the analysis of the experimental dependence shown in Figure 10, it was concluded that as the cutting tool wear power high-frequency components of the machine spindle oscillation increases relative power of low-frequency components. This can be taken as a basis for choosing a blunt cutting tool test.

The results will form the basis of the general concept phasechronometric system that can be built into the design of machine tools. The functions of the system are the diagnostics of technical condition of machine components and cutting tool wear monitoring

\section{References}

1. J. Lee, B. Bagheri, H. Kao, E. Lapira, Manuf. Leadership Journ., 02, (2015).

2. D.T. Matt, E. Rauch, Industriemanagement 01, (2015)

3. M. Kiselev and V. Pronyakin, Meas. Techn. 9, (2001)

4. A.B. Syritskii, Meas. Techn. 6, (2016)

5. A.S. Komshin, Meas. Techn. 8, (2013)

6. M.I. Kiselev [and others], Meas. Techn. 9, (2011)

7. K. Potapov, A. Syritskii, Pribory. 5, (2014)

8. D. Boldasov, N. Lazarev, A. Syritskii, Pribory. 10, (2015) 